\title{
Microfluidics: A Tool to Control the Size and Composition of Particles
}

\author{
Esther Amstad*
}

\begin{abstract}
Particles and capsules are used as containers for active ingredients to delay their degradation and control the location and kinetics of their release. Key to a successful application of these containers is a good control over their size, composition, and the release kinetics of encapsulants. These parameters can be tuned if containers are made from drops of a controlled size and composition; a method that enables formation of drops of a defined size is microfluidics. This review highlights some recent developments in the use of drops made with microfluidics to produce particles and capsules of controlled sizes, compositions, and structures.
\end{abstract}

Keywords: Encapsulation · Microfluidics

\section{From Drops to Particles}

Drops are often employed as templates for the production of particles with diameters ranging from a few nanometers up to millimeters. These particles are used as carriers of active substances for a wide range of applications including pharmacy, ${ }^{[1]}$ food, ${ }^{[2]}$ cosmetics, ${ }^{[3]}$ fragrances, ${ }^{[4]}$ and agriculture. ${ }^{[5]}$ Most applications require good control over the release kinetics of the encapsulated actives. The release kinetics can be tuned with the dimensions of the particles, which scale with those of the drops. The degree of control over the size of drops depends on the type of drops and their production method. There are two different types of drops that are most commonly employed to produce particles: Drops surrounded by air, which are used to fabricate dry particles, and drops surrounded by a second, immiscible liquid, which are used to fabricate particles dispersed in solutions.

Airborne drops can be produced in spray-dryers which are often used in industry to produce dry powders because they achieve high throughputs and are cost effective. ${ }^{[6]}$ They form airborne drops by pushing a solution through a nozzle

${ }^{\star}$ Correspondence: Prof. Dr. E. Amstad Soft Materials Laboratory, Institute of Materials Ecole Polytechnique Fédérale de Lausanne (EPFL) EPFL-STI-IMX-SMAL

Station 12

$\mathrm{CH}-1015$ Lausanne

E-mail: esther.amstad@epfl.ch where it is nebulized. The solvent is subsequently dried such that dry particles result. However, the control over the size of the resulting particles is limited and their diameter is usually well above $1 \mu \mathrm{m}$. Much smaller particles, with diameters below 500 $\mathrm{nm}$ can be formed in microfluidic spraydryers, albeit at much lower throughputs. ${ }^{[7]}$ Using these devices, it is even possible to control the structure of particles by tuning the processing conditions. ${ }^{[7 a, 8]}$ However, to allow for a more widespread use of these devices, further adjustments in their design and processing condition are needed to facilitate their operation and increase their throughput.

Much more work has been dedicated to simultaneously control the size and structure of particles produced from emulsion drops, which are drops surrounded by an immiscible liquid.[9] A technique that offers a tight control over the size of drops, and therefore also over that of particles, is microfluidics. ${ }^{[10]}$ The first part of this review highlights recent developments in the use of these drops to produce polymeric particles and capsules of defined sizes. The second part of the review summarizes recent advancements in the use of double emulsion drops to control the composition and structure of one type of capsule, vesicles, which comprise very thin shells.

\section{Solid Particles Made from Emulsion Drops}

The size of drops produced in microfluidic devices can be conveniently tuned between $10 \mu \mathrm{m}$ and $200 \mu \mathrm{m}$ although drops with diameters down to $1 \mu \mathrm{m}$ can be made. ${ }^{[11]}$ Drops are converted into particles, by solidifying their content. ${ }^{[9 b, 12]}$ This is most often achieved by covalently linking reagents contained in the drops. Often employed solutes are monomers or oligomers with chemically reactive groups, such as acrylates or methacrylates, which are solidified through living free radical polymerizations..$^{[12 c, 13]}$ Alternatively, drops have been loaded with mixtures of thioland vinylsulfone-containing solutes ${ }^{[14]}$ or mixtures of thiol- and acryl-containing solutes $^{[15]}$ that are solidified through Michael additions. The latter reactions are more benign to cells and thus preferred for the production of cell-loaded hydrogel microparticles. ${ }^{[14]}$

Drops can also be converted into particles by ionically crosslinking polymers contained in them. The vast majority of work on ionically crosslinked microparticles produced from drops has been performed on alginate, which can be crosslinked with $\mathrm{Ca}^{2+}$ ions. ${ }^{[16]}$ If an alginate-containing solution is mixed with a $\mathrm{Ca}^{2+}$-containing solution inside a microfluidic device immediately before drops are formed, alginate tends to gel so rapidly that it clogs microfluidic channels, which leads to the failure of these devices. [16b] To overcome this difficulty, the crosslinking kinetics must be reduced. This can be achieved if alginate is mixed with $\mathrm{CaCO}_{3}$ particles. The low solubility of $\mathrm{CaCO}_{3}$ in aqueous solutions at $\mathrm{pH}>7$ prevents its dissolution such that there are only very few free $\mathrm{Ca}^{2+}$ ions in solution. These few ions are insufficient to generate a percolating network of alginate, such that the solution remains fluid and does not clog microfluidic channels. If drops containing a mixture of alginate and $\mathrm{CaCO}_{3}$ are collected in acidified oil, the $\mathrm{pH}$ inside the drops decreases such that the $\mathrm{CaCO}_{3}$ solubility increases. The dissolved $\mathrm{Ca}^{2+}$ ions gel alginate, resulting in homogeneously crosslinked alginate hydrogels. ${ }^{[17]}$ Similar results can be achieved if $\mathrm{Ca}^{2+}$ ions are complexed, for example with ethylene diamine tetraacetic acid 
(EDTA). Solutions containing mixtures of alginate and CaEDTA can be controllably broken into drops that are collected in acidified oil. When the $\mathrm{pH}$ in the drops decreases, the affinity of $\mathrm{Ca}^{2+}$ to EDTA decreases such that a significant fraction of $\mathrm{Ca}^{2+}$ ions dissociates and the dissociated ions subsequently gel alginate. ${ }^{[18]}$ However, changes in $\mathrm{pH}$ can be detrimental to cells, hampering many biological applications. To avoid pH-shifts, CaEDTA complexes have been mixed with complexes encompassing a cation, whose affinity to EDTA is higher than that of $\mathrm{Ca}^{2+}$ and whose affinity to its chelate is lower than that to EDTA. For example, $\mathrm{Zn}^{2+}$ has a higher affinity to EDTA than $\mathrm{Ca}^{2+}$ and a lower affinity to ethyl diamine diacetate (EDDA) than to EDTA. If $\mathrm{Zn}^{2+}$ is initially complexed with EDDA and subsequently mixed with a solution containing CaEDTA, it replaces $\mathrm{Ca}^{2+}$ to form ZnEDTA complexes such that the free $\mathrm{Ca}^{2+}$ can gel alginate. This ion exchange is sufficiently slow to initiate crosslinking of alginate only after drops have been collected, thereby mitigating the risk for microfluidic channels to clog. [19]

Drops can also be solidified by physically crosslinking solutes. This can be achieved through solvent extraction, if the solvent has some solubility in the surrounding phase and thus partitions into it before it evaporates from the liquid-air interface. Thereby, the solute concentration in the drop steadily increases until it reaches its saturation concentration, where solutes start to precipitate. The precipitates are pulled together by the interfacial tension force of the shrinking drop and, when the vast majority of the solvent is evaporated, solid particles are formed.[20] This method is often employed to produce, for example, poly(lactide-co-glycolide) (PLGA) particles of defined sizes.

Independent of the method by which drops are solidified, the resulting particles are usually spheres whose diameter scales with that of the drops and the initial solute concentration. ${ }^{[12 b, 21]}$ The structure of these particles closely resembles that of bulk materials and is not influenced by the confinement or by the liquid-liquid interfaces. As a result, even though the kinetic properties, such as the swelling rate, depend on their size, the equilibrium properties of these particles closely resemble those of bulk samples. ${ }^{[13 c]}$

\subsection{Capsules Made from Single Emulsion Drops}

Capsules composed of a shell that encompasses a liquid or gaseous core can be made from single emulsion drops even if they are initially homogeneous. Probably the simplest capsules that can be formed from single emulsion drops are those whose shell is composed of a monolayer of amphiphiles that self-assemble at the liquid-liquid interface. These amphiphiles form a monolayer that stabilizes the emulsion drop such that the structure of the capsule shell is well defined. For example, amphiphiles composed of a perfluoropolyether chain containing a carboxylic acid head group, whose average molecular weight is $2.5 \mathrm{kDa}$, self-assemble at the liquid-liquid interface at room temperature in less than $1 \mathrm{~s}$, as shown schematically in Fig. 1a. ${ }^{[22]}$ This makes the formation of such capsules convenient and fast such that they are often used for screening assays. [23] However, these capsules are mechanically weak because adjacent amphiphiles only attract each other through weak Van-derWaals forces, thereby making these capsules susceptible to rupture if mechanically stressed. ${ }^{[24]}$ Moreover, these capsules often leak even in the absence of any external trigger: Low molecular weight additives are transported between different capsules, resulting in cross-contaminations, even if the encapsulants have no tangible solubility in the surrounding media. ${ }^{[25]}$ The leakage rate correlates with the surfactant concentration, suggesting that excessive surfactants contained in the surrounding oil form inverse micelles that transport encapsulants across the oil.[26] This transport is detrimental for screening assays because it results in cross-talk between the drops. It can be suppressed if the formation of inverse micelles is inhibited.
It has been shown that the formation of inverse micelles may be suppressed if the emulsion drops are stabilized with triblock copolymers composed of two blocks of perfluoroether (PFPE) that are interspaced by a polyglycerol block, which is much more hydrophilic and bulkier than PEG. ${ }^{[30]}$ However, it remains to be shown if these surfactants efficiently suppress the exchange of encapsulants between individual drops, even if drops are densely packed. This leakiness can be completely suppressed if drops are stabilized with nanoparticles that possess an appropriate wettability in the aqueous and oil phase. ${ }^{[31]}$ However, adsorption of nanoparticles at the liquid-liquid interface is slow such that the formation of drops exclusively stabilized with nanoparticles in microfluidic devices is more difficult compared to the formation of surfactant-stabilized drops.

The mechanical stability of surfactantstabilized drops increases and their permeability decreases if surfactants are made from polymers that exhibit stronger intermolecular forces. For example, if a hydrogen-bond acceptor, such as poly(vinyl pyrrolidone) (PVP), is adsorbed at the liquid-liquid interface and subsequently exposed to a liquid containing an hydrogen-bond donor, such as poly(methacrylic acid) (PMA), the two polymers interact with each other through strong hydrogenbonds. The resulting capsules are mechanically much more stable ${ }^{[32]}$ and display a

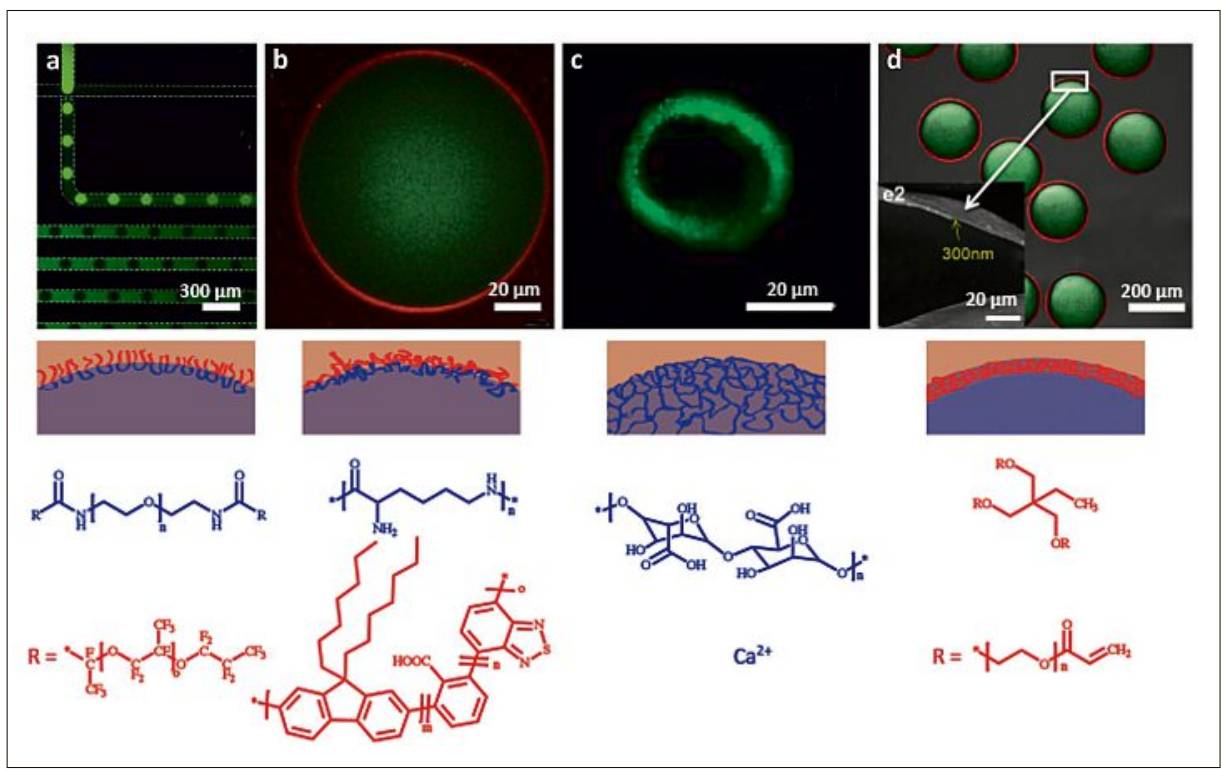

Fig. 1. Capsules made from single emulsion drops. Fluorescent micrographs of capsules whose shell is composed of (a) a monolayer of self-assembled block-copolymers (adapted from ref. [26a] with permission of Nature Publishing group); (b) coacervates formed between the positively charged poly(L-lactic acid) (PLL) and the negatively charged poly(fluorene-co-benzothiadiazoleco-benzoic acid) (adapted from ref. [27] with permission of The Royal Society of Chemistry); (c) ionically crosslinked alginate (adapted from ref. [28] with permission from American Chemical Society) and (d) covalently crosslinked trimethylolpropane ethoxylate triacrylate (ETPTA) formed in a drop that underwent phase separation (adapted from ref. [29] with permission of The Royal Society of Chemistry). The structures of the shells of the capsules are schematically illustrated in the middle panel, their chemical structures are shown at the bottom. 
significantly lower permeability towards low molecular weight encapsulants. They thus retain volatile additives, such as perfumes, for an extended time. [33]

A similar assembly strategy can be employed to form capsules composed of ionically interacting polymers. In this case, one of the polyelectrolytes is dissolved in the oil phase and the other in the aqueous phase. The oppositely charged polyelectrolytes attract each other and assemble at the liquid-liquid interface, even if they are not surface active, thereby forming capsules with thin shells, as schematically exemplified in Fig. 1b. ${ }^{[27,34]}$ These capsules display excellent mechanical stability and low permeability. ${ }^{[35]}$ However, the shells are heterogeneous because oppositely charged polyelectrolytes form small precipitates that are trapped at the liquid-liquid interface, by analogy to coacervates formed in bulk. ${ }^{[36]}$ It is possible to reduce the number of defects in these shells by increasing the number of polyelectrolyte layers to six. This is achieved by alternatingly dragging a drop through solutions containing the cationic and anionic polyelectrolytes. ${ }^{37]}$ However, the quality of the resulting polyelectrolyte multilayer capsule, its structure and properties remain to be determined.

Capsules with much thicker shells can be made if one of the reagents is soluble in both phases. In this case, reagents do not only interact at the liquid-liquid interface but one of them can penetrate into the other phase. For example, $\mathrm{Ca}^{2+}$ ions, initially dissolved in the oil as $\mathrm{CaI}_{2}$, can penetrate into aqueous drops. If these drops contain alginate, $\mathrm{Ca}^{2+}$ ions will initially gel the alginate molecules, which are closest to the liquid-liquid interface. Thereby, a thin shell composed of gelled alginate forms. If the resulting capsules are kept in this oil, $\mathrm{Ca}^{2+}$ ions continue to diffuse into the fluid core to gel additional alginate molecules, thereby increasing the thickness of the capsule shell. If the capsules are transferred into a $\mathrm{Ca}^{2+}$-free solution before all of the alginate is crosslinked, the gelation is arrested, resulting in capsules with thick shells and liquid cores, as schematically shown in Fig. 1c. [28]

Capsules with thicker shells can also be made from single emulsion drops, if all the reagents are contained in the drop. This is possible if the liquid contained in the drop phase separates over time and the interfacial tension between the first phase and the surrounding liquid is significantly lower than that between the second phase and the surrounding liquid. If these two conditions are met, the first phase spreads along the drop surface and engulfs the second phase, thereby spontaneously forming double emulsions. ${ }^{[38]}$ If reagents are preferentially contained in the first phase, they can be so- lidified, resulting in capsules whose shell thickness depends on the initial composition of the drop and can vary between 200 $\mathrm{nm}$ and $100 \mu \mathrm{m}$, as exemplified in Fig. 1d. ${ }^{[29]}$ However, this process is delicate and the number of solvents, solutes, and encapsulants that can be employed is very small. And even if all these conditions are met, this process offers very limited control over the structure of the shell. Thus, alternative strategies to controllably form capsules that offer more freedom in the choice of the composition of the solvents and wall materials and therefore allow tuning the capsule properties more accurately are usually preferred.

\subsection{Capsules Made from Double Emulsion Drops}

The number of materials that can be used to produce capsules is much larger if they are made from double emulsion drops, which are drops encompassing smaller drops. Double emulsion drops whose shell thickness varies between $5 \mu \mathrm{m}$ and a few 10 s of $\mu \mathrm{m}$ can be controllably made with microfluidics. ${ }^{[39]}$ They are converted into capsules by solidifying their shell. The thickness of the resulting capsule shells scales with that of the double emulsion drops and varies between $80 \mathrm{~nm}^{[9 \mathrm{a}, 40,41]}$ and a few 10s of $\mu \mathrm{m} .{ }^{[9 a, 42]}$ Because these capsules are made from three different fluids, encapsulants can be loaded into them at efficiencies close to $100 \%$, which is much higher than what is achieved for capsules produced through bulk emulsification processes.

The vast majority of capsules produced from double emulsions have shells with thicknesses exceeding $5 \mu \mathrm{m}$. The structure and properties of these shells closely resemble those of the corresponding bulk material. ${ }^{[42 b, 43]}$ These capsules are well suited for applications that require burst release, which occurs when the capsules suddenly become defective or if they rupture. ${ }^{[42 \mathrm{a}, 44]}$ These capsules are also suited for applications that require a continuous release of encapsulants. This can be achieved if encapsulates diffuse through the capsule shell[42b,45] or if capsule shells slowly disintegrate. ${ }^{[9 c, 34 c, 46]}$ However, these capsules do not allow controlled reversible changes in their permeability, which would be required for pulsed releases.

\section{Vesicles}

Nature produces capsules with very well defined structures whose permeability can be selectively and reversibly changed. A prime example are cell membranes, which are composed of bilayers of lipids. They can be made selectively permeable towards certain solutes by incorporating transmembrane proteins. These proteins can reversibly change the selective permeability of membranes, for example, if certain ligands bind to them or if electrical potentials are applied. ${ }^{[47]}$

Capsules whose structure closely mimics cell membranes are vesicles made of lipids, so-called liposomes. They are usually self-assembled in bulk through re-hydration methods ${ }^{[48]}$ or electroformation. ${ }^{[49]}$ However, because their shell is very thin and the attractive forces between adjacent lipids are weak, they are usually too fragile to be used as capsules. Vesicles can be made mechanically much more stable if they are composed of block-copolymers; these vesicles are called polymersomes. ${ }^{[50]}$ For block-copolymers to self-assemble into vesicular structures, the cross-sections of the hydrophilic and the hydrophobic blocks must be similar. ${ }^{[48 c, 51]}$ Within this limit, the structure and composition of the block-copolymers can be adjusted to tune the mechanical stability and permeability of the polymersomes. ${ }^{[50 \mathrm{~b}]}$

The permeability of polymersomes depends on the degree of hydrophobicity of their membrane core. Polymersomes with a less hydrophobic membrane core display a higher permeability. For example, polymersomes made of Pluronics L121, a poly(ethylene oxide)-poly(propylene oxide)-poly(ethylene oxide) (PEO-PPOPEO) block-copolymer are highly permeable. ${ }^{[52]}$ Their permeability can be reduced if a block-copolymer containing a strongly hydrophobic block, such as PEGpoly(butadiene) (PEG-PBD) is incorporated into their membrane. ${ }^{[53]}$ The permeability of polymersomes can be decreased even more if they are composed solely of block-copolymers with strongly hydrophobic blocks, such as PEG-PBD ${ }^{[52]}$ or PEG-poly(styrene) (PEG-PS). ${ }^{[54]}$ Indeed, the permeability of polymersomes made of PEG-PS towards $\mathrm{H}_{2} \mathrm{O}$ is so low, that they attain non-spherical shapes if subjected to osmotic pressures that they retain over days. [54]

To allow for a triggered release of encapsulants, the permeability of capsules must change if subjected to external stimuli. This can be achieved if responsive additives are incorporated into their membrane. ${ }^{[55]}$ Polymersomes are composed of self-assembled block-copolymers with a well-defined structure such that additives can self-assemble into this structure in a defined orientation. For example, polymersome membranes can be functionalized with transmembrane proteins, even though their membrane is significantly thicker than that of liposomes. These proteins render polymersomes reversibly permeable to selected solutes. ${ }^{[56]}$ However, the number of stimuli such polymersomes respond to is limited to those used by nature. 
Polymersomes can be made responsive to a wide variety of stimuli, if they are functionalized with stimuli-responsive block-copolymers. Polymersomes containing high concentrations of responsive block-copolymers tend to disintegrate if subjected to changes in the corresponding stimuli such as $\mathrm{pH},{ }^{[57]}$ temperature, ${ }^{[58]} \mathrm{UV}$ light, ${ }^{[58]}$ or oxidative environments. ${ }^{[59]}$ This prevents a slow continued or a repeated pulsed release of encapsulants.

To make polymersome membranes mechanically more robust, thereby preventing their disintegration, adjacent block-copolymers can been crosslinked. ${ }^{[60]}$ However, crosslinking prevents the diffusion of most encapsulants through the membrane, thereby precluding their release. A possibility to enable slow release from these mechanically stable polymersomes is to functionalize them with small pores, for instance, by incorporating phospholipids into the polymersome membrane. These phospholipids phase-separate and form small domains before adjacent block-copolymers are crosslinked. The phospholipids can subsequently be removed with detergents, resulting in pores, through which encapsulants can diffuse. ${ }^{[61]}$ An attractive alternative to pores is domains that display a high permeability to certain encaspulants. Domains form if polymersomes are made from mixtures of block-copolymers that phase-separate. ${ }^{[62]}$ The size of these domains depends on the exact composition of each vesicle. However, the composition of different vesicles made through selfassembly in bulk varies, even if they are produced in the same batch. As a result, the domains have different sizes, ${ }^{[62 a, b, 63]}$ limiting the control over the size of encapsulants that can be released and their release kinetics.

\subsection{Microfluidic Production of Vesicles}

Vesicles with very small variances in their composition can be produced from water-oil-water double emulsion drops. This method does not rely on the self-assembly of amphiphiles in bulk. Instead, it relies on their self-assembly at liquid-liquid interfaces. In this case, polymersomes are formed by dissolving amphiphiles in the oil phase prior to the formation of double emulsions, as schematically shown in Fig. 2a. These amphiphiles self-assemble at the two liquid-liquid interfaces of double emulsion drops to form two monolayers, as schematically illustrated in Fig. $2 \mathrm{~b}$. If the shell of the double emulsion is very thin such that the areas of the two liquid-liquid interfaces are similar, they associate upon removal of the majority of the oil, resulting in vesicles, as schematically shown in Figs $2 b$ and 2c. ${ }^{[66]}$ Whether these vesicles are completely free of oil remains to be shown. However, their mechanical properties closely resemble those of the corresponding vesicles made through rehydration methods, which are free of any oil residues, indicating that there is very little, if any oil left in the shell.[64]

\subsection{Production of Double Emulsions with Thin Shells}

Double emulsion drops with shell thicknesses below $1 \mu \mathrm{m}$ are difficult to obtain in a controlled way. It can be achieved in microfluidic glass capillary devices, which consist of an injection capillary and a collection capillary that are both inserted into a square capillary. ${ }^{[66 a, b, d, 67]}$ If the microfluidic devices are well-aligned and appropriately surface treated, double emulsions with shell thicknesses down to $800 \mathrm{~nm}$ can be formed. ${ }^{[66 \mathrm{~d}]}$ However, the production and operation of these devices is difficult because it relies on the formation of a thin oil film that flows along the walls of the injection capillary and encompasses the inner aqueous phase, as shown schematically in Fig. 3a and in the optical micrograph in Fig. $3 b$. If the thin oil film breaks because capillaries are misaligned, the surface treatment is insufficient, or if the fluid flow rates deviate from the optimum values, the inner aqueous phase contacts the outermost aqueous phase and single emulsion drops form. This problem can be alleviated if the inner fluid is broken into plugs at the junction where the inner and the middle phase meet, as shown in the optical micrograph in Fig. 3c. ${ }^{[66 d]}$ However, this operation furnishes a mixture of single and double emulsion drops that must be separated after their production. Moreover, it results in a broader distribution of shell thicknesses. Nevertheless, it is the most often used mode to produce double emulsions with shell thicknesses below $1 \mu \mathrm{m}$.

The reproducible fabrication of microfluidic devices is much easier if they are made from masks or molds, such that all of them have identical dimensions. Soft lithography is often employed to produce microfluidic devices that are predominantly made from poly(dimethyl siloxane) (PDMS). ${ }^{[68]}$ To mimic the three-dimensional structure of glass capillary devices, channels are incorporated into each of the two pieces of PDMS that are subsequently bonded to each other. To ensure good alignment of the two channel-containing PDMS pieces, one piece contains pins and the other one contains holes such that the two PDMS pieces can be slid into each other using the lock and key mechanism. ${ }^{[69]}$ This simple but powerful feature facilitates the production of well-aligned devices and therefore increases their yield to close to $100 \%$. Unfortunately, it is much more difficult to tune the wettability of surfaces made of PDMS than those made of glass. Therefore, the oil films that flows along the walls of PDMS channels must be thicker than in glass to prevent their disintegration. This translates into thicker shells of the resulting double emulsions, as shown in Fig. 3d. Indeed, the shell thickness of double emulsions made in PDMS devices could not be reduced below $5 \mu \mathrm{m},{ }^{[70]}$ which is too large to convert them into vesicles. The shell thickness can be reduced down to $300 \mathrm{~nm}$ if double emulsions are subjected to fast-flowing fluids ${ }^{[71]}$ or pushed through constrictions, as shown in the time-laps

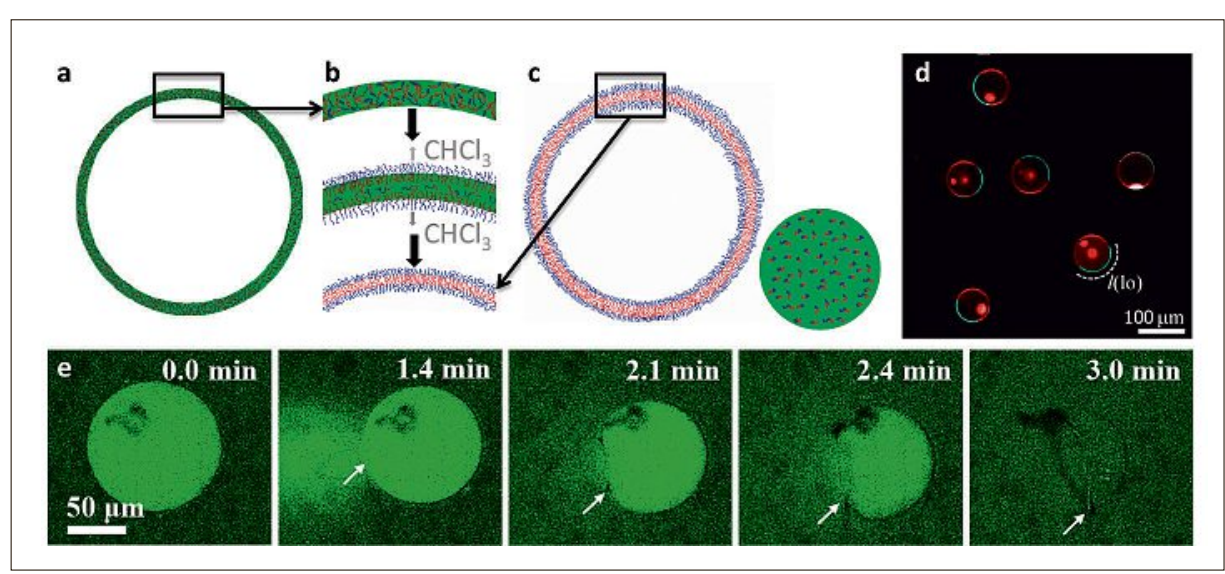

Fig. 2. (a-c) Schematic illustration of the formation of polymersomes from double emulsion drops. (a) A water-oil-water double emulsion drop with the amphiphilic block-copolymers dispersed in the oil phase. (b) The amphiphilic block-copolymers self-assemble at the liquid-liquid interfaces to form two monolayers. When the oil separates from the double emulsion shell, the two monolayers associate to form a (c) polymersome and a single emulsion oil drop that contains excessive block-copolymers. (d) Fluorescence micrograph of liposomes formed from double emulsion drops. Liposomes are composed of a mixture of lipids that phase-separate, resulting in two different domains that are labeled with red and green dyes respectively. Reproduced from ref. [64] with permission of Wiley- $\mathrm{VCH}$ ). (e) Time-lapse fluorescence micrographs of thermo-responsive polymersomes that has been made light-responsive by incorporating gold nanoparticles in its membrane (reprinted from ref. [65] with permission of Wiley-VCH). 


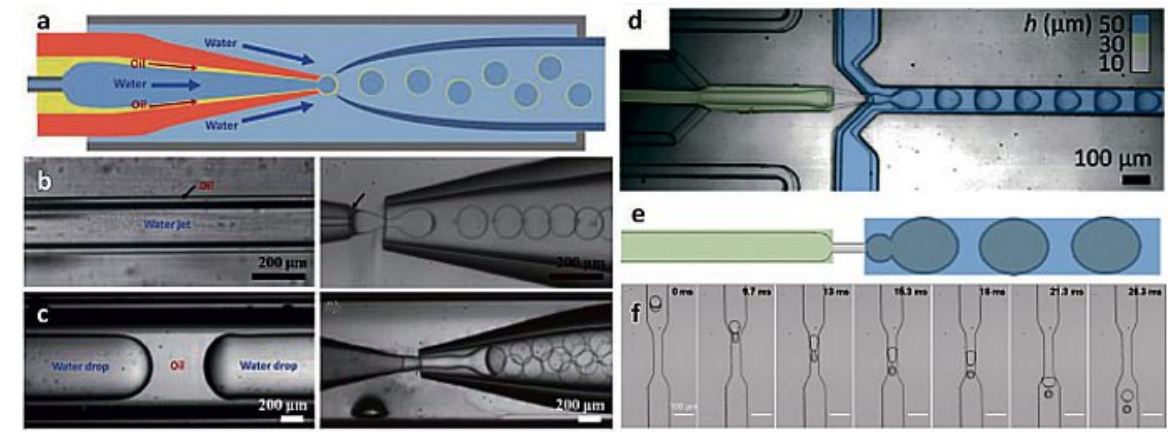

Fig. 3. (a) Schematic illustration and (b,c) optical micrograph of a microfluidic glass capillary device that produces double emulsions with thin shells. The innermost aqueous phase forms a (b) jet and (c) plugs in the injection capillary (left), before double emulsion drops are formed (right). Reprinted from ref. [66d] with permission of The Royal Society of Chemistry. (d) Top view optical micrograph and (e) schematic illustration of the side view of a PDMS microfluidic device that forms double emulsions with thin shells. Reprinted from ref. [70] with permission of The Royal Society of Chemistry. (f) Time-lapse optical micrographs of a double emulsion that is pushed through a constriction to reduce its shell thickness from $5 \mu \mathrm{m}$ to $300 \mathrm{~nm}$. Reprinted from ref. [72] with permission of Springer.

optical micrographs in Fig. 3e. ${ }^{[70,72]}$ These methods do not impose restrictions on the choice of oils and are thus much more versatile. However, it remains to be shown if further adjustments on the microfluidic device design allow complete removal of oil from these double emulsion shells and hence open new possibilities to convert double emulsions into vesicles.

\subsection{Conversion of Double Emulsions into Vesicles}

To convert water-oil-water double emulsions into vesicles, the oil must be removed from their shell. This can be achieved if double emulsions are at the liquid-air interface such that the oil can evaporate. [73] However, the yield of the resulting polymersomes is low because many double emulsions rupture during the evaporation of the oil. To increase the yield, oil must be removed from double emulsions that are fully inserted in the surrounding liquid. ${ }^{[74]}$ This can be achieved if the oil is volatile and has some solubility in the aqueous phase, such that it partitions into it and subsequently evaporates from the water-air interface.[66a,c,71,75] Solvents that are partially soluble in aqueous solutions usually have a low water-solvent interfacial tension. However, to enable a spontaneous drop formation, without the need for external actuators, the interfacial tension between the oil and the outer aqueous phase must be sufficiently high. These partially contradictory requirements limit the number of oils that can be used for this application. Indeed, there are only three oil mixtures that have been reported to allow conversion of double emulsions into vesicles: combinations of chloroform and hexane, ${ }^{[71,76]}$ chloroform and toluene, ${ }^{[66 a, b]}$ and toluene and tetrahydrofurane (THF). ${ }^{[66 c]}$ However, these solvent mixtures are toxic and prevent most biological and biomedical applications. It remains to be seen if the new PDMS-based microfluidic devices can overcome this difficulty and enable the production of vesicles from biocompatible oils.

\subsection{Vesicles Produced from Double Emulsions}

The size of vesicles is very similar to that of double emulsion drops they are made from. Their membrane is made of a single bilayer of self-assembled amphiphiles. However, this bilayer is not perfect because there is always some excess of amphiphiles in the oil phase. These excessive amphiphiles are up-concentrated on one side of the double emulsion during the final stages of oil removal such that they form patches, thereby introducing defects into the resulting vesicle membrane.[66b,77] These defects prevent studies on the mechanical properties of cell-membrane mimicking vesicles. However, they can be advantageous for the use of vesicles as capsules because they make them mechanically more robust: The defects serve as reservoirs of amphiphiles, which can be used to enlarge the area of vesicle membranes if mechanically stressed or subjected to osmotic pressures, thereby preventing their rupture. If the concentration of excessive amphiphiles is even higher, the amphiphiles are distributed within the hydrophobic part of the bilayer. These amphiphiles spontaneously assemble into bilayers that bud off from the polymersome surface, to form many much smaller vesicles. ${ }^{78]}$

In a first approximation, the composition of vesicles produced from double emulsions resembles that of the amphiphiles added to the oil phase prior to the assembly of double emulsions. This enables the assembly of vesicles composed of different amphiphiles where the composition of each vesicle is the same, within experimental error. This is exemplified on vesicles composed of a mixture of lipids that is known to undergo phase separation: 1,2-dioleyl-sn-glycero-3-phosphocholine (DOPC), 1,2-dipalmitoyl-sn-glycero3-phosphocholine (DPPC), and cholesterol. ${ }^{[79]}$ These liposomes all have identical domain sizes if made from double emulsion drops, as shown in Fig. 2d.[64] This opens up possibilities to design domains composed of responsive amphiphiles that become leaky if exposed to external stimuli but are too small to cause rupture of the entire vesicle. ${ }^{[80]}$

The production of vesicles from double emulsion drops offers an additional benefit: It relies on the self-assembly of amphiphiles at liquid-liquid interfaces. Hence, the geometric requirements that must be met by them to be incorporated into vesicle membranes are less stringent than if produced through bulk self-assembly processes. This facilitates incorporation of block-copolymers that do not fulfill the steric requirements to form vesicular membranes into them. For example, membranes made of PEG-poly(lactic acid) (PEGPLA) can be made thermo-responsive if PEG(6 kDa)-PNIPAM(5.5 kDa) block-copolymers, whose ratio of radii of gyration of PEG:PNIPAM is 2.4, are incorporated into them. ${ }^{[65]}$ Similarly, membranes of Pluronics L121 can be made more leaky if functionalized with Pluronics L61, a blockcopolymer that does not self-assemble into vesicular structures. ${ }^{[52,74]}$ However, if the concentration of these block-copolymers is too high, they introduce defects into the membranes that are sufficiently large to cause their disintegration. ${ }^{[65]}$ This sets an upper limit to the amount of such functional block-copolymers that can be incorporated into membranes.

Additional functionality can be imparted to vesicles by adding nanoparticles into their membranes. ${ }^{[81]}$ This can be achieved by dispersing the nanoparticles in the oil prior to the formation of double emulsion drops. A large fraction of the nanoparticles remains in the hydrophobic part of the bilayer even after the oil is removed, thereby allowing the production of polymersomes loaded with high quantities of nanoparticles. ${ }^{[82]}$ For example, liposomes could be made responsive to magnetic fields by incorporating iron oxide nanoparticles into their membranes. ${ }^{[82]}$ Similarly, nonresponsive polymersomes could be made responsive to oxidative environments by incorporating manganese oxide nanoparticles into their membranes, ${ }^{[83]}$ and thermoresponsive polymersomes could be made light responsive by incorporating gold nanoparticles into their membranes, as shown in Fig. 3e. ${ }^{[65]}$ These examples dem- 
onstrate that it is possible to functionalize vesicles with nanoparticles that would be too large to self-assemble into vesicle membranes. ${ }^{[83]}$ However, protocols that offer a close control over the concentration of incorporated nanoparticles remain to be established.

\subsection{Current Limitations}

Most polymersomes produced from double emulsion templates have diameters between 50 and $100 \mu \mathrm{m}$ and are composed of PEG-PLA or PEG-PLGA block-copolymers. These polymersomes are fragile, making their handling difficult and hampering their use as capsules because they typically rupture within a few days up to a few weeks. The stability of these polymersomes can be increased if their membrane is made of two bilayers. ${ }^{[76 a]}$ However, this makes their assembly very difficult. Their stability can be increased even further if PLA homopolymers are incorporated into the bilayer. ${ }^{[84]}$ But even in this case, they tend to rupture if manipulated with syringes or if stored for prolonged times. Polymersomes are mechanically very robust if the hydrophobic part of their membrane is made of flexible polymers that do not crystallize. For example, polymersomes made of PMOXA-PDMS-PMOXA display a very high mechanical stability. ${ }^{[60 b, 85]}$ Even though polymersomes composed of PEGPDMS block-copolymers with diameters close to $100 \mu \mathrm{m}$ have been formed from double emulsion drop templates, the mechanical properties of these vesicles have never been quantified. ${ }^{[75]}$ Hence, it remains to be seen if mechanically robust polymersomes can be made from double emulsion drop templates.

If amphiphilic block-copolymers are assembled into polymersomes using double emulsion drops as templates, the polymers have two functions: They initially stabilize double emulsion drops and subsequently are the building blocks of the polymersome membrane. ${ }^{[24]}$ Unfortunately, the currently used amphiphilic block-copolymers such as PEG-PLA and PEG-PLGA impart a limited stability to double emulsion drops such that they tend to coalesce before the oil can be removed from their shell. To prevent coalescence, these drops are usually stabilized with additional surfactants, such as poly (vinyl alcohol) (PVA). However, there is strong evidence that PVA partially incorporates into the polymersome membranes, thereby changing their mechanical properties. ${ }^{[86]}$ Therefore, it would be important to exclude any additional surfactants during and after the formation of double emulsions. This has been shown for polymersomes composed of PEO(1.3 kDa)-b-PBD(2.5 kDa) by carefully collecting the double emulsion drops and slowly evaporating the oil from their shell.[87] However, this process is delicate and the yield of polymersomes is low. Hence, there is a need for new protocols that facilitate the conversion of double emulsions into vesicles and for new blockcopolymers that more efficiently stabilize double emulsion drops.

The production of polymersomes from double emulsions has important advantages because it allows close control over their size and offers encapsulation efficiencies close to $100 \%$. However, the volume that can be encapsulated per device and hour is limited to approximately $1 \mathrm{~mL}$. Even though feasibility to operate up to four devices in parallel to increase the throughput has been shown for glass capillary devices, ${ }^{[67]}$ and PDMS-based devices, ${ }^{[70]}$ up-scaling this technology still presents a major challenge. Overcoming this challenge requires new devices that form drops more robustly. A possibility to produce drops with a precise control over their size at a much higher throughput has recently been reported in PDMS ${ }^{[11 \mathrm{c}]}$ and glass devices. ${ }^{[88]}$ Whether this technology is also suitable for the fabrication of double emulsions with thin shells at such high throughputs remains to be shown.

\section{Conclusions}

Particles and capsules are often formed from drops by solidifying their contents. Methods that offer close control over the size of the drops, and therefore also over the size of the resulting particles are well established and broadly used by different communities. Similarly, much work has been devoted to establishing protocols that enable good control over the composition of these particles such that their properties can be tuned. By contrast, little attention has been paid to the structure of these particles and capsules despite its important influence on their properties. Initial steps to address this shortcoming have recently been undertaken with developments of protocols that offer superior control over the solidification kinetics of drops. However, additional work is needed to produce capsules that satisfy the increasingly demanding requirements on the control over their properties.

Vesicles are capsules with very well defined shell structures. They can be made from double emulsion drops, which offer tight control over their size, and composition. This technique is used especially for the production of functional polymersomes. However, the influence of the composition and structure of the block-copolymers on the properties of polymersomes made from double emulsion drops remains to be tested. There is only a small number of block-copolymers that have thus far been used to produce polymersomes from double emulsion drops. This might partially be attributed to the still incomplete understanding of the influence of the composition and structure of amphiphilic block-copolymers on their assembly at liquid-liquid interfaces and their ability to stabilize emulsion drops. These factors are crucial for the production of polymersomes from double emulsion drops. The limited understanding of the influence of the composition and structure of blockcopolymers on the properties of double emulsions and polymers currently hampers many applications of capsules with thin shells. A better understanding of this influence would open up new possibilities to design responsive capsules with thin shells and offer better control over their surface chemistries.

Received: April 12, 2017

[1] a) A. Singh, G. Van den Mooter, $A d v$. Drug Deliv. Rev. 2016, 100, 27; b) L. Chen, T. Okuda, X. Y. Lu, H. K. Chan, Adv. Drug Deliv. Rev. 2016, 100, 102.

[2] a) C. Encina, C. Vergara, B. Gimenez, F. Oyarzun-Ampuero, P. Robert, Trends in Food Science \& Technol. 2016, 56, 46; b) A. Goncalves, B. N. Estevinho, F. Rocha, Trends in Food Science \& Technol. 2016, 51, 76.

[3] F. Casanova, L. Santos, J. Microencapsulation 2016, 33, 1 .

[4] S. Ghayempour, M. Montazer, J Microencapsulation 2016, 33, 497.

[5] P. L. Kashyap, X. Xiang, P. Heiden, Int. J. Biol. Macromol. 2015, 77, 36.

[6] P. Schuck, R. Jeantet, B. Bhandari, X. D. Chen, I. T. Perrone, A. F. de Carvalho, M. Fenelon, P. Kelly, Drying Technol. 2016, 34, 1773.

[7] a) E. Amstad, M. Gopinadhan, C. Holtze, C. O. Osuji, M. P. Brenner, F. Spaepen, D. A. Weitz, Science 2015, 349, 956; b) E. Amstad, F. Spaepen, D. A. Weitz, J. Phys. Chem. B 2016, 120, 9161; c) J. Thiele, M. Windbergs, A. R. Abate, M. Trebbin, H. C. Shum, S. Forster, D. A. Weitz, Lab on a Chip 2011, 11, 2362; d) E. Amstad, F. Spaepen, M. P. Brenner, D. A. Weitz, Lab on a Chip 2017.

[8] E. Amstad, F. Spaepen, D. A. Weitz, PCCP 2015, 17, 30158 .

[9] a) S. S. Datta, A. Abbaspourrad, E. Amstad, J. Fan, S.-H. Kim, M. Romanowsky, H. C. Shum, B. Sun, A. S. Utada, M. Windbergs, S. Zhou, D. A. Weitz, Adv. Mater. 2014, 26, 2205; b) W. J. Duncanson, T. Lin, A. R. Abate, S. Seiffert, R. K. Shah, D. A. Weitz, Lab on a Chip 2012, 12, 2135; c) W. Wang, M. J. Zhang, L. Y. Chu, Acc. Chem. Res. 2014, 47, 373.

[10] a) A. S. Utada, L. Y. Chu, A. Fernandez-Nieves, D. R. Link, C. Holtze, D. A. Weitz, Mrs Bulletin 2007, 32, 702; b) P. Garstecki, I. Gitlin, W. DiLuzio, G. M. Whitesides, E. Kumacheva, H. A. Stone, Appl. Phys. Lett. 2004, 85, 2649; c) P. B. Umbanhowar, V. Prasad, D. A. Weitz, Langmuir 2000, 16, 347; d) S. L. Anna, N. Bontoux, H. A. Stone, Appl. Phys. Lett. 2003, 82,364 .

[11] a) W.-C. Jeong, J.-M. Lim, J.-H. Choi, J.-H Kim, Y.-J. Lee, S.-H. Kim, G. Lee, J.-D. Kim, G.-R. Yi, S.-M. Yang, Lab on a Chip 2012, 12, 1446; b) C. Cohen, R. Giles, V. Sergeyeva, N Mittal, P. Tabeling, D. Zerrouki, J. Baudry, J. Bibette, N. Bremond, Microfluidics and 
Nanofluidics 2014, 17, 959; c) E. Amstad, M. Chemama, M. Eggersdorfer, L. R. Arriaga, M. Brenner, D. A. Weitz, Lab on a Chip 2016, 16, 4163.

[12] a) D. Dendukuri, P. S. Doyle, Adv. Mater. 2009, 21, 4071; b) S. Seiffert, Angew. Chem. Int. Ed. 2013, 52, 11462; c) E. Tumarkin, E. Kumacheva, Chem. Soc. Rev. 2009, 38, 2161.

[13] a) S. Seiffert, J. Thiele, A. R. Abate, D. A Weitz, J. Am. Chem. Soc. 2010, 132, 6606; b) F. Di Lorenzo, S. Seiffert, Macromol. React. Eng. 2016, 10, 201; c) S. Seiffert, Macromol. Rapid Commun. 2012, 33, 1135

[14] a) S. Allazetta, L. Kolb, S. Zerbib, J. Bardy, M. P. Lutolf, Small 2015, 11, 5647; b) S. Allazetta, T. C. Hausherr, M. P. Lutolf, Biomacromolecules 2013, 14, 1122.

[15] T. Rossow, J. A. Heyman, A. J. Ehrlicher, A. Langhoff, D. A. Weitz, R. Haag, S. Seiffert, J. Am. Chem. Soc. 2012, 134, 4983.

[16] a) K. Liu, H. J. Ding, J. Liu, Y. Chen, X. Z. Zhao, Langmuir 2006, 22, 9453; b) L. Mazutis, R. Vasiliauskas, D. A. Weitz, Macromol. Biosci. 2015, 15, 1641.

[17] M. Marquis, V. Alix, I. Capron, S. Cuenot, A. Zykwinska, Acs Biomater. Sci. Eng. 2016, 2, 535.

[18] S. Utech, R. Prodanovic, A. S. Mao, R. Ostafe, D. J. Mooney, D. A. Weitz, Adv. Healthcare Mater. 2015, 4, 1628

[19] a) A. G. Hati, N. B. Arnfinnsdottir, C. Ostevold, M. Sletmoen, G. Etienne, E. Amstad, B. T. Stokke, RCS Adv. 2016, 6, 114830; b) A. G. Hati, D. C. Bassett, J. M. Ribe, P. Sikorski, D. A. Weitz, B. T. Stokke, Lab on a Chip 2016, 16, 3718.

[20] a) A. Perez, R. Hernandez, D. Velasco, D Voicu, C. Mijangos, J. Coll. Interface Sci. 2015, 441, 90; b) K. Keohane, D. Brennan, P. Galvin, B. T. Griffin, Int. J. Pharm. 2014, 467, 60; c) P. B. Odonnell, J. W. McGinity, Adv. Drug Deliv. Rev. 1997, 28, 25.

[21] W. J. Duncanson, T. E. Kodger, S. Babaee, G. Gonzalez, D. A. Weitz, K. Bertoldi, Langmuir 2015, 31, 3489.

[22] B. Riechers, F. Maes, E. Akoury, B. Semin, P. Gruner, J. C. Baret, PNAS 2016, 113, 11465.

[23] a) E. Brouzes, M. Medkova, N. Savenelli, D. Marran, M. Twardowski, J. B. Hutchison, J. M. Rothberg, D. R. Link, N. Perrimon, M. L. Samuels, PNAS 2009, 106, 14195; b) J. J. Agresti, E. Antipov, A. R. Abate, K. Ahn, A. C. Rowat, J. C. Baret, M. Marquez, A. M. Klibanov, A. D. Griffiths, D. A. Weitz, PNAS 2010, 107, 4004; c) M. T. Guo, A. Rotem, J. A. Heyman, D. A. Weitz, Lab on a Chip 2012, 12, 2146.

[24] G. Etienne, M. Kessler, E. Amstad, Macromol. Chem. Phys. 2016, 210, DOI: 10.1002/ macp.201600365.

[25] P. Gruner, B. Riechers, L. A. Chacòn Orellana, Q. Brosseau, F. Maes, T. Beneyton, D. Pekin, J.-C. Baret, Curr. Opin. Colloid Interface Sci. $2015,20,183$

[26] a) P. Gruner, B. Riechers, B. Semin, J. Lim, A. Johnston, K. Short, J. C. Baret, Nat. Comm. 2016, 7, 10392; b) Y. Skhiri, P. Gruner, B. Semin, Q. Brosseau, D. Pekin, L. Mazutis, V. Goust, F. Kleinschmidt, A. El Harrak, J. B. Hutchison, E. Mayot, J. F. Bartolo, A. D. Griffiths, V. Taly, J. C. Baret, Soft Matter 2012, 8, 10618 .

[27] H. Monteillet, F. Hagemans, J. Sprakel, Soft Matter 2013, 9, 11270.

[28] H. Zhang, E. Tumarkin, R. Peerani, Z. Nie, R. M. A. Sullan, G. C. Walker, E. Kumacheva, $J$ Am. Chem. Soc. 2006, 128, 12205.

[29] N. N. Deng, W. Wang, X. J. Ju, R. Xie, D. A. Weitz, L. Y. Chu, Lab on a Chip 2013, 13, 4047.

[30] O. Wagner, J. Thiele, M. Weinhart, L. Mazutis, D. A. Weitz, W. T. S. Huck, R. Haag, Lab on a Chip 2016, 16, 65.
[31] a) M. Pan, L. Rosenfeld, M. Kim, M. Xu, E Lin, R. Derda, S. K. Y. Tang, ACS Appl. Mater. Interfaces 2014, 6, 21446; b) M. Pan, M. Kim, L. Blauch, S. K. Y. Tang, RCS Adv. 2016, 6, 39926.

[32] S. Le Tirilly, C. Tregouet, M. Reyssat, S. Bone, C. Geffroy, G. Fuller, N. Pantoustier, P. Perrin, C. Monteux, Langmuir 2016, 32, 6089.

[33] S. Le Tirilly, C. Tregouet, S. Bone, C. Geffroy, G. Fuller, N. Pantoustier, P. Perrin, C. Monteux, Acs Macro Lett. 2015, 4, 25.

[34] a) T. Y. D. Tang, C. R. C. Hak, A. J. Thompson, M. K. Kuimova, D. S. Williams, A. W. Perriman, S. Mann, Nature Chem.2014, 6, 527; b) T Wang, N. Y. He, Nanoscale 2010, 2, 230; c) A. P. Esser-Kahn, S. A. Odom, N. R. Sottos, S. R. White, J. S. Moore, Macromolecules 2011, 44, 5539.

[35] a) D. S. Hwang, H. B. Zeng, A. Srivastava, D. V. Krogstad, M. Tirrell, J. N. Israelachvili, J. H. Waite, Soft Matter 2010, 6, 3232; b) C. G. de Kruif, F. Weinbreck, R. de Vries, Curr Opin. Colloid Interface Sci. 2004, 9, 340; c) M. Kim, S. J. Yeo, C. B. Highley, J. A. Burdick, P. J. Yoo, J. Doh, D. Lee, Acs Nano 2015, 9, 8269; d) G. Kaufman, R. Boltyanskiy, S. Nejati, A. R. Thiam, M. Loewenberg, E. R. Dufresne, C. O. Osuji, Lab on a Chip 2014, 14, 3494.

[36] G. Dardelle, P. Erni, Adv. Colloid Interface Sci. 2014, 206, 79 .

[37] a) C. Kantak, S. Beyer, L. Yobas, T. Bansal, D. Trau, Lab on a Chip 2011, 11, 1030; b) C. Priest, A. Quinn, A. Postma, A. N. Zelikin, J. Ralston, F. Caruso, Lab on a Chip 2008, 8, 2182.

[38] T. Y. Lee, T. M. Choi, T. S. Shim, R. A. M. Frijns, S. H. Kim, Lab on a Chip 2016, 16, 3415 .

[39] a) A. S. Utada, E. Lorenceau, D. R. Link, P. D Kaplan, H. A. Stone, D. A. Weitz, Science 2005, 308, 537; b) S. Okushima, T. Nisisako, T. Torii, T. Higuchi, Langmuir 2004, 20, 9905.

[40] a) A. Abbaspourrad, N. J. Carroll, S. H. Kim, D. A. Weitz, J. Am. Chem. Soc. 2013, 135, 7744; b) A. Abbaspourrad, S. S. Datta, D. A. Weitz, Langmuir 2013, 29, 12697.

[41] B. F. B. Silva, C. Rodriguez-Abreu, N. Vilanova, Curr. Opin. Colloid Interface Sci. 2016, 25, 98.

[42] a) E. Loiseau, A. Q. de Boiry, F. Niedermair, G. Albrecht, P. A. Ruhs, A. R. Studart, Adv. Funct Mater. 2016, 26, 4007; b) P. W. Chen, R. M Erb, A. R. Studart, Langmuir 2012, 28, 144.

[43] a) P. W. Chen, G. Cadisch, A. R. Studart, Langmuir 2014, 30, 2346; b) P. W. Chen, J. Brignoli, A. R. Studart, Polymer 2014, 55, 6837.

[44] a) S. Neuser, P. W. Chen, A. R. Studart, V. Michaud, Adv. Eng. Mater. 2014, 16, 581; b) M. H. Lee, K. C. Hribar, T. Brugarolas, N. P. Kamat, J. A. Burdick, D. Lee, Adv. Funct. Mater. 2012, 22, 131.

[45] M. A. Zieringer, N. J. Carroll, A. Abbaspourrad, S. A. Koehler, D. A. Weitz, Small 2015, 11, 2903.

[46] a) B. Kim, T. Y. Jeon, Y. K. Oh, S. H. Kim, Langmuir 2015, 31, 6027; b) T. S. Shim, S H. Kim, S. M. Yang, Particle \& Particle Sys. Charact. 2013, 30, 9.

[47] a) D. X. Fu, A. Libson, L. J. W. Miercke, C. Weitzman, P. Nollert, J. Krucinski, R. M Stroud, Science 2000, 290, 481; b) I. Horváth, G. Multhoff, A. Sonnleitner, L. Vígh, Biochim. Biophys. Acta (BBA) - Biomembranes 2008 , 1778,1653 ; c) A. J. Brake, M. J. Wagenbach, D. Julius, Nature 1994, 371, 519; d) R. O. Fox, F. M. Richards, Nature 1982, 300, 325

[48] a) R. C. Macdonald, R. I. Macdonald, B. P. M. Menco, K. Takeshita, N. K. Subbarao, L. R. Hu, Biochim. Biophys. Acta 1991, 1061, 297; b) C. Kirby, G. Gregoriadis, Bio-Technology 1984, 2, 979; cD. E. Discher, A. Eisenberg, Science 2002, 297, 967.
[49] a) M. I. Angelova, D. S. Dimitrov, Faraday Discuss. 1986, 81, 303; b) M. I. Angelova, S. Soleau, P. Meleard, J. F. Faucon, P. Bothorel, 'Preparation of Giant Vesicles by External AC Electric-Fields - Kinetics and Applications', Vol. 89, 1992.

[50] a) J. F. Le Meins, O. Sandre, S. Lecommandoux, Eur. Phys. J. E 2011, 34; (b) D. E. Discher, F. Ahmed, in 'Annual Review of Biomedical Engineering', Vol. 8, 2006, p. 323.

[51] S. Jain, F. S. Bates, Science 2003, 300, 460.

[52] R. Rodriguez-Garcia, M. Mell, I. LopezMontero, J. Netzel, T. Hellweg, F. Monroy, Soft Matter 2011, 7, 1532

[53] L. Yan, E. Higbee, A. Tsourkas, Z. L. Cheng, J. Mater. Chem. B 2015, 3, 9277.

[54] R. S. M. Rikken, H. Engelkamp, R. J. M. Nolte, J. C. Maan, J. C. M. van Hest, D. A. Wilson, P. C. M. Christianen, Nat. Comm. 2016, 7, 12606.

[55] a) J. Gaitzsch, D. Appelhans, L. G. Wang, G. Battaglia, B. Voit, Angew. Chem. Int. Ed. 2012, 51, 4448; b) C. G. Palivan, R. Goers, A. Najer, X. Y. Zhang, A. Car, W. Meier, Chem. Soc. Rev. 2016, 45, 377; c) M. H. Li, P. Keller, Soft Matter 2009, 5, 927.

[56] a) P. Broz, S. Driamov, J. Ziegler, N. Ben-Haim, S. Marsch, W. Meier, P. Hunziker, Nano Lett. 2006, 6, 2349; b) M. Kumar, M. Grzelakowski, J. Zilles, M. Clark, W. Meier, PNAS 2007, 104, 20719; (c) C. Nardin, S. Thoeni, J. Widmer, M. Winterhalter, W. Meier, Chem. Commun. 2000, 1433.

[57] K. T. Kim, J. Cornelissen, R. J. M. Nolte, J. C. M. van Hest, Adv. Mater. 2009, 21, 2787.

[58] C. Z. Yao, X. R. Wang, G. H. Liu, J. M. Hu, S. Y. Liu, Macromolecules 2016, 49, 8282.

[59] A. Napoli, M. Valentini, N. Tirelli, M. Muller, J. A. Hubbell, Nat. Mater. 2004, 3, 183.

[60] a) J. Gaitzsch, D. Appelhans, D. Graefe, P. Schwille, B. Voit, Chem. Commun. 2011, 47, 3466; b) C. Nardin, T. Hirt, J. Leukel, W. Meier, Langmuir 2000, 16, 1035.

[61] Z. L. Cheng, A. Tsourkas, Langmuir 2008, 24, 8169.

[62] a) K. Spinler, A. W. Tian, D. A. Christian, D. A. Pantano, T. Baumgart, D. E. Discher, Langmuir 2013, 29, 7499; b) S. A. Meeuwissen, S. M. C. Bruekers, Y. Chen, D. J. Pochan, J. C. M. van Hest, Polymer Chem. 2014, 5, 489; c) C. LoPresti, M. Massignani, C. Fernyhough, A. Blanazs, A. J. Ryan, J. Madsen, N. J. Warren, S. P. Armes, A. L. Lewis, S. Chirasatitsin, A. J. Engler, G. Battaglia, Acs Nano 2011, 5, 1775; d) G. Xue, K. Chen, G. Shen, Z. Wang, Q. Zhang, J. Cai, Y. Li, Colloids Surf. A Physicochem. Eng. Asp. 2013, 436, 1007.

[63] a) D. A. Christian, A. Tian, W. G. Ellenbroek, I. Levental, K. Rajagopal, P. A. Janmey, A. J. Liu, T. Baumgart, D. E. Discher, Nat. Mater. 2009, 8, 843; b) M. Chemin, P. M. Brun, S. Lecommandoux, O. Sandre, J. F. Le Meins, Soft Matter 2012, 8, 2867.

[64] L. R. Arriaga, S. S. Datta, S.-H. Kim, E. Amstad, T. E. Kodger, F. Monroy, D. A. Weitz, Small 2014, 10, 950.

[65] E. Amstad, S.-H. Kim, D. A. Weitz, Angew. Chem. Int. Ed. 2012, 12499.

[66] a) H. C. Shum, D. Lee, I. Yoon, T. Kodger, D. A. Weitz, Langmuir 2008, 24, 7651; b) H. C. Shum, J.-W. Kim, D. A. Weitz, J. Am. Chem. Soc. 2008, 130, 9543; c) T. Foster, K. D. Dorfman, H. T. Davis, J. Coll. Interface Sci. 2010, 351, 140; d) S.-H. Kim, J. W. Kim, J.C. Cho, D. A. Weitz, Lab on a Chip 2011, 11, 3162.

[67] S. H. Kim, J. W. Kim, D. H. Kim, S. H. Han, D. A. Weitz, Microfluidics and Nanofluidics 2013, 14, 509.

[68] Y. N. Xia, G. M. Whitesides, Ann. Rev. Mater. Sci. 1998, 28, 153.

[69] a) A. Rotem, A. R. Abate, A. S. Utada, V. Van Steijn, D. A. Weitz, Lab on a Chip 2012, 12, 
4263; b) F. C. Chang, Y. C. Su, J. Micromech. Microeng. 2008, 18, 065018.

[70] L. R. Arriaga, E. Amstad, D. A. Weitz, Lab on a Chip 2015, 15, 3335.

[71] H. C. Shum, E. Santanach-Carreras, J.-W. Kim, A. Ehrlicher, J. Bibette, D. A. Weitz, J. Am. Chem. Soc. 2011, 133, 4420

[72] A. Vian, V. Favrod, E. Amstad, Microfluidics and Nanofluidics 2016, 20, 159.

[73] T. Foster, K. D. Dorfman, H. T. Davis, Langmuir 2010, 26, 9666.

[74] D. F. do Nascimento, L. R. Arriaga, M. Eggersdorfer, R. Ziblat, M. d. F. V. Marques, F. Reynaud, S. A. Koehler, D. A. Weitz, Langmuir 2016, 32, 5350 .

[75] R. C. Hayward, A. S. Utada, N. Dan, D. A. Weitz, Langmuir 2006, 22, 4457.

[76] a) S. H. Kim, J. Nam, J. W. Kim, D. H. Kim, S. H. Han, D. A. Weitz, Lab on a Chip 2013, 13, 1351; b) H. C. Shum, Y. J. Zhao, S. H. Kim,
D. A. Weitz, Angew. Chem. Int. Ed. 2011, 50, 1648.

[77] Y. D. Hu, J. Perez-Mercader, Colloids Surf. B 2016, 146, 406.

[78] J. Thiele, V. Chokkalingam, S. H. Ma, D. A. Wilson, W. T. S. Huck, Mater. Horiz. 2014, 1, 96.

[79] S. L. Veatch, I. V. Polozov, K. Gawrisch, S. L. Keller, Biophys. J. 2004, 86, 2910.

[80] O. Sandre, L. Moreaux, F. Brochard-Wyart, PNAS 1999, 96, 10591

[81] a) E. Amstad, J. Kohlbrecher, E. Mueller, T. Schweizer, M. Textor, E. Reimhult, Nano Lett. 2011, 11, 1664; b) E. Amstad, E. Reimhult, Nanomedicine 2012, 7, 145; c) Y. J. Liu, B. Liu, Z. H. Nie, Nano Today 2015, 10, 278.

[82] A. Seth, G. Bealle, E. Santanach-Carreras, A. Abou-Hassan, C. Menager, Adv. Mater. 2012, 24,3544 .

[83] R. W. Jaggers, R. Chen, S. A. F. Bon, Mater. Horiz. 2016, 3, 41.
[84] C. Martino, S. H. Kim, L. Horsfall, A. Abbaspourrad, S. J. Rosser, J. Cooper, D. A. Weitz, Angew. Chem. Int. Ed. 2012, 51, 6416.

[85] A. Graff, M. Sauer, P. Van Gelder, W. Meier, PNAS 2002, 99, 5064.

[86] W.-S. Jang, S. C. Park, M. Kim, J. Doh, D. Lee, D. A. Hammer, Macromol. Rapid Commun. 2015, 36, 378 .

[87] N. P. Kamat, M. H. Lee, D. Lee, D. A. Hammer, Soft Matter 2011, 7, 9863.

[88] A. Ofner, D. G. Moore, P. A. Rühs, P. Schwendimann, M. Eggersdorfer, E. Amstad, D. A. Weitz, A. R. Studart, Macromol. Chem. Phys. 2017, 218, UNSP 1600472. 